\title{
Implementação de uma liga acadêmica de sistematização de enfermagem na região Norte
}

\author{
Implementation of an academic league of systematization of nursing assistance in the \\ North
}

\begin{abstract}
Implementación de una liga académica de sistematización de la asistencia de enfermería en el Norte
\end{abstract}

Jaqueline Dantas Neres Martins ${ }^{1 *}$, Beatriz Duarte de Oliveira ${ }^{1}$, Stephany Siqueira Braga ${ }^{1}$, Ana Alice Matias Ambé ${ }^{2}$, Carmem Lúcia Pacheco de Sena ${ }^{1}$, Roseli Reis da Silva ${ }^{3}$, Flávia Maia Araripe ${ }^{2}$, Daniele Melo Sardinha ${ }^{4}$, Milena Farah Damous Castanho Ferreira ${ }^{2}$, Marcelo Williams Oliveira de Souza ${ }^{5}$.

\section{RESUMO}

Objetivo: Relatar a experiência de acadêmicos do Curso de Graduação em Enfermagem na implementação da Liga Acadêmica de Sistematização da Assistência de Enfermagem (LASAE) na cidade de Belém do Pará. Relato de experiência: Sobre a trajetória de implementação da LASAE, a mesma foi fundada no dia 09 de janeiro de 2019, por um grupo de 06 acadêmicos do curso de Graduação em Enfermagem, com o objetivo de discutir temáticas relacionadas à Sistematização da Assistência de Enfermagem (SAE), Processo de Enfermagem $(\mathrm{PE})$, teorias de enfermagem, bem como aprimorar conhecimentos relacionados à padronização e nomenclaturas de diagnósticos e intervenções em enfermagem. As atividades realizadas promoveram contribuição na formação de estudantes de enfermagem e desenvolvimento de habilidades e qualidades fundamentais na prática profissional, assim como na valorização da SAE e PE, como ferramentas de trabalho e imprescindíveis para a cientificidade profissional. Considerações finais: A implementação da liga acadêmica proporcionou a ampliação do conhecimento relacionado a SAE e PE, além de ter contribuído na diminuição das deficiências e lacunas não esclarecidas na universidade. Foi demonstrado também, boa recepção das atividades promovidas pela LASAE.

Palavras-chave: Processo de enfermagem, Enfermagem, Conhecimento, Educação em enfermagem.

\section{ABSTRACT}

Objective: To report the experience of undergraduate nursing students in the implementation of the Academic League for Nursing Care Systematization (LASAE) in the city of Belém do Pará. Experience report: About LASAE's implementation trajectory, it was founded on January 9, 2019, by a group of 06 undergraduate Nursing students, with the aim of discussing topics related to the Systematization of Nursing Assistance (SAE), Nursing Process (PE), nursing theories, as well as how to improve knowledge related to the standardization and nomenclatures of nursing diagnoses and interventions. The activities carried out promoted a contribution to the training of nursing students and the development of fundamental skills and qualities in professional practice, as well as in the valuation of SAE and PE, as work tools and essential for professional scientificity. Final considerations: The implementation of the academic league provided the expansion of the knowledge related to $\mathrm{SAE}$ and $\mathrm{PE}$, besides contributing to the reduction of deficiencies and gaps not clarified in the university. It was also demonstrated, good reception of the activities promoted by LASAE.

Keywords: Nursing process, Nursing, Knowledge, Nursing education.

\footnotetext{
1 Universidade do Estado do Para (UEPA), Belém - PA. *E-mail: jaqueline170896@gmail.com

${ }^{2}$ Centro Universitário Metropolitano da Amazônia (UNIFAMAZ), Belém - PA.

${ }^{3}$ Faculdade Pan Amazônica (FAPAN), Belém - PA.

${ }^{4}$ Instituto Evandro Chagas (IEC), Belém - PA.

5Universidade Federal do Pará (UFPA), Belém - PA.
} 


\section{RESUMEN}

Objetivo: Informar sobre la experiencia de los estudiantes de pregrado de enfermería en la implementación de la Liga Académica para la Sistematización del Cuidado de Enfermería (LASAE) en la ciudad de Belém do Pará. Informe de la experiencia: Sobre la trayectoria de implementación de LASAE, fue fundada el 9 de enero de 2019, por un grupo de 06 estudiantes de enfermería de pregrado, con el objetivo de discutir temas relacionados con la Sistematización de la Asistencia de Enfermería (SAE), el Proceso de Enfermería (PE), las teorías de enfermería, así como cómo mejorar el conocimiento relacionado con la estandarización y nomenclaturas de diagnósticos e intervenciones de enfermería. Las actividades realizadas promovieron una contribución a la formación de estudiantes de enfermería y al desarrollo de habilidades y cualidades fundamentales en la práctica profesional, así como en la valoración de SAE y PE, como herramientas de trabajo y esenciales para la cientificidad profesional. Consideraciones finales: La implementación de la liga académica proporcionó la ampliación del conocimiento relacionado a SAE y PE, además de haber contribuido en la disminución de las deficiencias y lagunas no esclarecidas en la universidad. Se ha demostrado también, buena recepción de las actividades promovidas por LASAE.

Palabras clave: Proceso de enfermería, Enfermería, Conocimiento, Educación en enfermería.

\section{INTRODUÇÃO}

A sistematização da assistência de enfermagem (SAE) é o método científico que direciona e organiza a dinâmica de trabalho do enfermeiro, promovendo maior autonomia na prática assistencial, assim como uma assistência segura e de qualidade ao paciente. A SAE é aplicável a qualquer instância e ambiência de atividade laboral do enfermeiro, podendo se fazer presente em diferentes vertentes dos processos de cuidado, por meio de protocolos, fluxogramas, sistemas de informação, escalas de funcionários, bem como pela operacionalização do Processo de Enfermagem (PE) (LINCH GFC, et al., 2019).

Com a Resolução 358/2009 do Conselho Federal de Enfermagem (COFEN) elucida-se e normatiza o PE (Processo de Enfermagem) como método de execução da SAE, instituindo o mesmo como uma atividade privativa do enfermeiro, subsidiado por etapas inter-relacionadas, interdependentes auxiliando o enfermeiro em sua tomada de decisão, na adoção de medidas resolutivas e na avaliação de sua assistência (COFEN, 2009).

Nesse ínterim, ao fortalecer os conhecimentos acerca da SAE permite-se capacitar enfermeiros para atender as demandas e necessidades de seu paciente, corroborando para a promoção de uma prática científica, segura e de qualidade, viabilizando um perfil estratégico direcionado a conduzir seu processo de trabalho de forma organizada, dinamizada e sistemática, plausível aos valores, atributos, competências e habilidades regulamentadas pelas diretrizes curriculares e normatizadas pela Lei do Exercício Profissional 7.498/86 e pela Resolução 358/2009 do COFEN (LINCH GFC, et al., 2019; CONFEN, 1986).

Contudo, estudos demonstram que a SAE ainda possui desafios na sua implementação, evidenciados principalmente pela deficiência na abordagem da temática durante o curso de graduação; assim como barreiras burocráticas e administrativas, falta de pessoal e recursos materiais para assistência; falta de articulação entre a teoria e a prática; sensibilização acerca da temática; além das influências do modelo biomédico no cuidado (GUTIÉRREZ MGR e MORAIS SCRV, 2017).

Diante disso, as Diretrizes Curriculares Nacionais do Curso de Graduação em Enfermagem com o intuito de regulamentar e orientar requisitos para a formação de profissionais enfermeiros demonstra grande preocupação com as ações, valores, conhecimentos e habilidades destes profissionais em seus ambientes de trabalho, tornando de conhecimento público o perfil ideal para que tais atributos e valores sejam alcançados, ressaltando a prática de sistematizar a sua assistência como algo imprescindível e parte da sua tomada de decisão (BRASIL, 2001).

Desse modo, entende-se que há uma necessidade em fortalecer tal temática no processo de formação e capacitação do profissional enfermeiro, sendo a implantação de uma Liga Acadêmica de Sistematização de Assistência de Enfermagem um elo fundamental na consolidação desse processo. Visto que as ligas 
acadêmicas visam proporcionar ao acadêmico reflexão crítica e raciocínio científico sobre determinada temática, desenvolvendo conhecimento teórico-prático ao acadêmico no âmbito do tripé universitário; ensino, pesquisa e extensão (SILVA SA e FLORES O, 2015).

Logo, o presente trabalho tem o objetivo de relatar a experiência de acadêmicos do Curso de Graduação em Enfermagem na implementação da Liga Acadêmica de Sistematização da Assistência de Enfermagem, na cidade de Belém do Pará.

\section{RELATO DE EXPERIÊNCIA}

Sobre a trajetória de implementação da Liga Acadêmica de Sistematização da Assistência de Enfermagem (LASAE), a mesma foi fundada no dia 9 de janeiro de 2019, por um grupo de 6 acadêmicos (diretores) do curso de Graduação em Enfermagem, com o objetivo de discutir temáticas relacionadas à SAE, PE, teorias de enfermagem, bem como aprimorar conhecimentos relacionados à padronização e nomenclaturas de diagnósticos e intervenções em enfermagem.

Inicialmente, nos meses de janeiro e fevereiro houve encontros com os membros fundadores para discutir sobre o funcionamento, criação do Estatuto e o planejamento das atividades da liga acadêmica. Em março de 2019, a liga vinculou-se a uma Instituição de Ensino Superior, na qual se efetivou a reunião quinzenal aos sábados pela manhã.

A LASAE apresenta os seus princípios e competências embasadas nas Diretrizes Curriculares Nacionais do Curso de Graduação em Enfermagem, entre elas: Atenção a Saúde, tomada de decisões, comunicação e liderança. A liga tem como base o tripé universitário ensino-pesquisa-extensão com a finalidade de promover maior aplicabilidade, inserção e vivência da SAE pelos graduandos em formação. A fim de favorecer e impulsionar o processo de aprendizado, a LASAE adota para a administração das aulas teóricas o uso de metodologias ativas, com o objetivo de fortalecer e anexar os conhecimentos obtidos.

Dessa forma, como atividade de ensino, houve capacitação teórico-prática sobre SAE aos membros diretores, ministrada pelo orientador geral nos meses de março, abril e maio de 2019. A capacitação foi ministrada com o auxílio de metodologias ativas, na qual utilizou-se material audiovisual com conteúdos impulsores de reflexão e instigação sobre a prática de enfermagem. Para a parte prática foram utilizados casos clínicos para debate. Estas aulas foram essenciais para estabelecer uma reflexão sobre o protagonismo do profissional de enfermagem e instigar ainda mais o interesse pela temática.

Como atividades de pesquisa, houve a criação de grupos de trabalhos para dar ênfase em produção de artigos científicos, produção de materiais e tecnologias leves de auxílio para as atividades de extensão, produção de trabalhos para serem apresentados em eventos científicos com o objetivo de oferecer devolutivas sobre as pesquisas e achados científicos. Assim como, a apresentação de trabalhos em congressos com o intuito de despertar o interesse do público acadêmico pela LASAE.

Além de cumprir as atividades de ensino e pesquisa, a LASAE promoveu dois eventos importantes e de grande valia para a comunidade acadêmica e os recentes membros efetivos no primeiro semestre de 2019: O primeiro foi o minicurso sobre SAE, etapas do PE e seus desafios na contemporaneidade, ministrada pelo coordenador geral da liga. Este evento foi dedicado ao público externo e de caráter complementar para o processo seletivo para ingresso na liga. Aos aprovados no Processo seletivo, houve o oferecimento da aula inaugural ministrado por convidada externa, na qual pautou sobre a temática: O enfermeiro, a SAE e a Lei.

Sobre a organização estrutural da LASAE: é composta por diretoria, coordenação, orientadores, membros colaboradores e membros efetivos. A diretoria é formada por estudantes de enfermagem que planejam e impulsionam as atividades da liga, nela contém 10 integrantes distribuídos da seguinte maneira: Presidente, vice-presidente, secretária, diretoria financeira, diretoria de ensino, diretoria de pesquisa, diretoria de extensão, diretoria social e de comunicação.

A coordenação, orientação e membros colaboradores são compostos por enfermeiros que tenham expertise na área de SAE e PE, responsáveis por auxiliarem no impulsionamento das programações estabelecidas e firmadas pelo conselho geral, assim como subsidiam a operacionalização do ensino, pesquisa e extensão. 
Dentre um dos aspectos positivos da implementação da LASAE encontra-se o pioneirismo, já que é a primeira liga no estado do Pará destinada a contemplar, discutir e desenvolver atividades relacionadas às ferramentas de trabalho da enfermagem, seus instrumentos metodológicos e rede de nomenclaturas e padronizações da enfermagem que conferem cientificidade a profissão e contribuem para a qualidade da assistência.

Entre as atividades propostas pela LASAE, a capacitação teórico-prática de Sistematização da Assistência de Enfermagem (entre os diretores da liga) viabilizou o debate e a explanação de assuntos imprescindíveis como: os desafios para a implementação da SAE e as teorias de Enfermagem como alicerce de cuidados. Nesse momento, os acadêmicos obtiveram a oportunidade de expressar suas dificuldades frente a temática, discutindo ampla e abertamente acerca de suas limitações e principalmente, debateram maneiras de como contorná-las utilizando o conhecimento científico como pilar.

Como estratégia para impulsionar o processo de ensino-aprendizagem, essa capacitação ocorreu mediante a utilização de metodologia ativa, com recursos audiovisuais ilustrativos, sobre situações rotineiras e mecanicistas que dificultam a implementação do PE nas instituições de saúde, mediante essas ilustrações foram incitadas perguntas e reflexões críticas sobre a enfermagem na contemporaneidade. Ao término da teorização da SAE e PE, houve a inclusão de casos clínicos para exercitar e fixar os conteúdos explicitados.

Visando a proliferação de conhecimento a LASAE proporcionou um minicurso de SAE aberto ao público de enfermagem, onde foram abordados conceitos primordiais para uma assistência efetiva e resolutiva, os assuntos elencados, foram desde os mais simples, explanando o que é a SAE e as etapas do Processo de Enfermagem, estes inclusos na mesma, até assuntos mais complexos, havendo grande interação entre os participantes do minicurso, assim como, a estimulação do pensamento crítico e de uma prática baseada em evidencias.

Ainda no viés da multiplicação de conhecimento e para atuar na realidade a qual estamos inseridos, a liga acadêmica é parceira de uma Unidade Básica de Saúde, onde serão desempenhadas atividades de Sistematização da Assistência de Enfermagem em prol e de acordo com as demandas dos usuários do serviço, assim como, atividades de educação em saúde e educação permanente com os funcionários da UBS. Ações como essa são pertinentes, visto que, os acadêmicos se familiarizam com atividades que os mesmos irão desempenhar como futuros enfermeiros e são capazes de transformar realidades através de suas ações frente à assistência ou como gestores e líderes de uma equipe.

Desta maneira, este conjunto de atividades e encontros promovidos e desempenhados pela LASAE, contribuíram na fixação de conhecimento, em especial daqueles relacionados a implementação do $P E$, como método científico. Assim como a construção e o desenvolvimento de habilidades fundamentais para 0 graduando, como, por exemplo, a tomada de decisões, aprimoramento das relações interpessoais e comunicação, trabalho em equipe, planejamento e liderança.

\section{DISCUSSÃO}

A liga acadêmica é um espaço de autonomia dos estudantes, onde há o firmamento e objetividade de aprimorar conhecimentos em determinadas temáticas nos eixos de ensino- pesquisa- extensão, na qual geralmente são conteúdos pouco nivelados na graduação. Considerando também que a díade espaçooportunidade oferecidas na Academia podem ser limitados e restritos diante da relevância e complexidade de tais temáticas (SILVA AS e FLORES O, 2015; SILVA JP, et al., 2015).

Portanto, é mister ressaltar que as diretrizes curriculares nacionais do curso de graduação em enfermagem enaltecem a importância da liga acadêmica na contribuição junto a formação de enfermeiros humanistas, críticos e reflexivos, comprometidos com as necessidades de saúde da população e empenhados sóciopoliticamente na melhora dos determinantes de saúde (BRASIL, 2001; GUTIÉRREZ MGR e MORAIS SCRV, 2017; SILVA JP, et al., 2015).

A constituição de uma liga acadêmica, portanto, influi positivamente em contribuições para a sociedade, pois é um espaço de construção e aprimoramento de conhecimento entre acadêmicos com o emprego de 
metodologias específicas voltadas para o melhor usufruto e fixação do conhecimento, na qual agrega valores e experiências. Sequencialmente, esses saberes incitam questionamentos e perguntas na qual conduzem a pesquisas destinadas a responder tal anseio. Por fim, a conquista desse conhecimento conduz a intervenções científicas que geram mudanças nos paradigmas e concepções sociais, e devolutivas imprescindíveis para a melhoria na comunidade, seja no aspecto da melhoria da qualidade de vida do paciente ou mesmo na melhora das práticas assistenciais (SILVA AS e FLORES O, 2015; SILVA JP, et al., 2015; CAVALCANTE ASP, et al., 2018).

Este contexto de criação de ligas acadêmicas voltadas a discussão da SAE e PE é relevante, uma vez que, segundo pesquisas há deficiência e lacunas de conhecimento na graduação sobre estas temáticas o qual favorece o egresso de um recém-graduado inapto ao exercício da enfermagem, fadado a prestar assistência despidas de rigor científico e favorável a cometer "erros". Essas são apenas alguns dos desfavores que podem ocorrer, mas ainda há outros que imputam na categoria profissional e promove desfecho desfavorável ao paciente e instituição de saúde (SILVA AS e FLORES O, 2015; SILVA JP, et al., 2015; BERALDO RAS e SOUZA NR, 2015; SOARES MI, et al., 2015).

Fato que comprova essa deficiência são as pesquisas que evidenciam as dificuldades de determinadas Instituições de saúde em implementar o PE em setores e clínicas. Estas pesquisas trazem como resultados que o empecilho de efetivação por vezes advém dos próprios enfermeiros, a qual reduzem e entendem a SAE como um impresso. Entre outros fatores estão a não adesão da equipe de enfermagem ao $P E$, não comparecimento a capacitação permanente promovida, mecanicismo, burocratização, priorização de outras atividades administrativas (BERALDO RAS e SOUZA NR, 2015; SOARES MI, et al., 2015).

No entanto, as consequências dessa não adesão pairam na descaracterização da profissão, assistência despida de cientificidade, desconformidade jurídico do enfermeiro em seguir suas atribuições e assim segue. Estas consequências tendem a ser cíclicas e gradualmente repercutem negativamente na qualidade da assistência (SILVA AS e FLORES O, 2015; ALENCAR IGM, et al., 2018; GIEHL CT, et al., 2016; RIEGEL F e JUNIOR NJO, 2017).

Esses aspectos são prioritariamente observados nas atividades da LASAE. Já que além de fornecer o suporte teórico, há grande preocupação em favorecer o processo ensino- aprendizado, mediante perguntas e questões os quais incitem os membros sobre a importância dessas ferramentas metodológicas no trabalho da enfermagem. As principais reflexões trazidas durante o semestre foram: A importância do PE para a cientificidade da profissão e as contribuições dessa aplicação para o Sistema Único de Saúde, reconhecimento profissional e qualidade, eficiência e seguridade do cuidado. Dentre outros aspectos ressaltados foram também a consonância da SAE junto aos demais programas institucionais e nacionais de melhora nas práticas assistenciais (RIEGEL F e JUNIOR NJO, 2017; RIBEIRO RG, et al., 2015; PEREIRA GN, et al., 2017).

Estes momentos de intercâmbio de conhecimento foram indispensáveis na constituição da liga e reformulação sobre os conteúdos já adquiridos na universidade. Particularmente, na maioria das graduações em enfermagem o ensino sobre SAE é reduzido a resolução de casos clínicos e aulas expositivas, tal enfoque minimiza a dimensão da SAE e não conduz ao desenvolvimento de raciocínio clínico (SILVA JP, et al., 2015; CAVALCANTE ASP, et al., 2018; RIBEIRO RG, et al., 2015).

É válido salientar que inseridas no conjunto de benefícios da liga acadêmica estão também o desenvolvimento de habilidades e aptidões imprescindíveis para o desenvolvimento profissional, como o aprimoramento da administração com o tempo, pessoas, desenvolvimento de liderança, expansão de oportunidades, capacidade de tomar decisões e discutir ideias. Estas qualidades são indispensáveis e requeridas no exercício profissional para a melhor condução das atividades junto ao paciente e equipe de saúde, visto que, o enfermeiro além de ser elo de conexão entre os demais profissionais é também o líder da equipe de enfermagem (RIBEIRO RG, et al., 2015; BERALDO RAS e SOUZA NR, 2015; SOARES MI, et al., 2015).

A LASAE trouxe ganhos à comunidade acadêmica uma vez, que há a necessidade de discutir e aprimorar o conhecimento relacionado à SAE e PE, visto que estas são os subsídios da prática da enfermagem e o 
embasamento para a cientificidade da profissão. As atividades foram imprescindíveis para a criação de espaço a qual discutissem as implicações legais da SAE e da atuação do enfermeiro; a anexação das teorias de enfermagem em tal contexto, os desafios e empecilhos à estruturação e efetivação de tais ferramentas na contemporaneidade. Estas discussões promoveram o intercâmbio de conhecimento e a autonomia de estudantes para organizar atividades fomentadas diante de uma das principais deficiências deixadas na graduação, que é o ensino sobre SAE e PE.

\section{REFERÊNCIAS}

1. ALENCAR IGM, et al. Implementação e Implantação da Sistematização da Assistência de Enfermagem. Rev. enferm. UFPE; 12 (4): 1174-8. DOI: https://doi.org/10.5205/1981-8963-v12i4a231030p1174-1178-2018

2. BERALDO RAS, SOUZA NR. Desafios na implantação da sistematização da assistência de enfermagem nos setores de urgência. Rev. enferm. UFPE on line; 9 (5):7773-80.

3. BRASIL. Ministério da Educação. Conselho Nacional de Educação. Resolução N 3, de 07 de novembro de 2001. Diretrizes curriculares nacionais do curso de graduação em Enfermagem.

4. CAVALCANTE ASP, et al. As Ligas Acadêmicas na Ảrea da Saúde: Lacunas do Conhecimento na Produção Científica Brasileira. Rev. bras. educ. méd. 2018.

5. CONSELHO FEDERAL DE ENFERMAGEM. Resolução 358/2009. Dispõe sobre a Sistematização da Assistência de Enfermagem e a implementação do Processo de Enfermagem em ambientes, públicos ou privados, em que ocorre o cuidado profissional de Enfermagem, e dá outras providências.

6. CONSELHO FEDERAL DE ENFERMAGEM. Lei 7.498, de 25 de junho 1986. Dispõe sobre a regulamentação do exercício da enfermagem e dá outras providências

7. GIEHL CT, et al. A equipe de enfermagem frente ao processo de implantação da Sistematização da Assistência de Enfermagem. Rev. enferm. atenção saúde. 2016; 5 (2): 87-95.

8. GUTIÉRREZ MGR, MORAIS SCRV. Systematization of nursing care and the formation of professional identity. Rev bras enferm. 2017 [Citado em 2019 jun 29]; 70 (2): 436-41. DOI: https://doi.org/10.1590/0034-7167-2016-0515.

9. LINCH GFC, et al. Ações coordenadas para implantação e consolidação da sistematização da assistência de enfermagem em um complexo hospitalar. Enferm. Foco. 2019; 19 (4): 82-8.

10. PEREIRA GN, et al. Relação entre Sistematização da Assistência de Enfermagem e segurança do paciente. Enferm. foco. 2017; 8 (2): 21-25.

11. RIEGEL F, JUNIOR NJO. Processo de enfermagem: implicações para a segurança do paciente em centro cirúrgico. Cogitare enferm. 2017; 22 (4).

12. RIBEIRO RG, et al. Systematization of nursing care: perception of nurses. Rev Enferm UFPI. $2015 ; 4$ (1): $47-53$.

13. SILVA JP, et al. Systematization of Nursing Care in undergraduate training: the perspective of Complex Thinking. Rev. latinoam. enferm. 2015; 23 (1): 59-66. DOI: https://doi.org/10.1590/0104-1169.0096.2525.

14. SILVA SA, FLORES O. Ligas Acadêmicas no Processo de Formação dos Estudantes. Rev Bras Educ Med. 2015; 39(3)410-417. DOI: https://doi.org/10.1590/1981-52712015v39n3e02592013.

15.SOARES MI, et al. Systematization of nursing care: challenges and features to nurses in the care management. Esc. Anna Nery Rev. Enferm. 2015; 19 (1): 47-53. 